\title{
Retrospective analysis of the efficacy and safety of rivaroxaban in the treatment of hepatic sinus obstruction syndrome caused by Gynura segetum
}

\section{Hao Bing}

First Affiliated Hospital of China Medical University

Dan Li

First Affiliated Hospital of China Medical University

Bing Chang

First Affiliated Hospital of China Medical University

Yiling Li ( $\sim$ liyiling127@163.com )

First Affiliated Hospital of China Medical University https://orcid.org/0000-0003-4143-9071

Xiangmin $\mathrm{He}$

First Affiliated Hospital of China Medical University

Jing Tong

First Affiliated Hospital of China Medical University

\section{Ying Wang}

First Affiliated Hospital of China Medical University

\section{Ran Ao}

First Affiliated Hospital of China Medical University

Ningning Wang

First Affiliated Hospital of China Medical University

\section{Research article}

Keywords: rivaroxaban; anticoagulant treatment; pyrrolidine alkaloids; Gynura segetum; hepatic sinusoidal obstruction syndrome

Posted Date: August 14th, 2019

DOI: https://doi.org/10.21203/rs.2.12785/v1

License: (c) (1) This work is licensed under a Creative Commons Attribution 4.0 International License. Read Full License 


\section{Abstract}

Background PA-HSOS is a rare disease and there is no specific treatment for PA-HSOS. Anticoagulant, antithrombotic and microcirculation therapy can alleviate the progression of PA-HSOS. The application of rivaroxaban in patients with PA-HSOS has not yet been reported. The aim of this study was to analyze the efficacy and safety of rivaroxaban in the treatment of pyrrolizidine alkaloid-induced hepatic sinusoidal obstruction syndromecaused by Gynura segetum (PA-HSOS). Methods A retrospective analysis was conducted with the clinical data of patients with PA-HSOS in the acute/subacute phase caused by taking Gynura segetum. The patients were divided into warfarin and rivaroxaban groups according to the anticoagulant therapy they received. Related biochemical indicators were monitored during hospitalization. Liver ultrasound, liver elastography and related biochemical indicators were reviewed every two weeks or one month after discharge. The patients were followed until 1 year after complete remission or death. The efficacy and safety of rivaroxaban was compared with that of warfarin according to the patients' hepatic venous recanalization rates and the occurrence of bleeding events. Results The study included 20 patients, with 10 patients in the warfarin group and 10 patients in the rivaroxaban group. The results showed that the average anticoagulant course in the rivaroxaban group was significantly shorter than that in the warfarin group $(P=0.007)$. With treatment, the remission rates of the rivaroxaban group and the warfarin group reached $90 \%$. There was no significant difference in the incidence of adverse reactions or bleeding events between the two groups $(P>0.05)$. Conclusions Compared with warfarin, rivaroxaban, a new oral anticoagulant, is convenient and safe for clinical use. It has an obvious effect on PA-HSOS and a low risk of bleeding. It provides a new anticoagulant treatment for PA-HSOS.

\section{Background}

Pyrrolizidine alkaloid-induced hepatic sinusoidal obstruction syndrome (PA-HSOS) refers to hepatic sinusoidal obstruction syndrome (HSOS) caused by the ingestion of plants containing pyrrolidine alkaloids (PAs). To date, more than 660 PAs and PA N-oxides have been identified in an estimated 6000 plants ${ }^{[1]}$; the most frequent herbal medicine that causes PA-HSOS reported in China is Gynura segetum, which is used to relieve pain, improve blood circulation, and dissipate blood stasis ${ }^{[2,3]}$. To date, the toxic mechanisms underlying PA-induced toxicity are not fully understood. Research shows that the metabolic activation of PAs is catalyzed by hepatic cytochrome P450 and generates reactive pyrrolic metabolites that bind to cellular proteins to form pyrrole-protein adducts leading to PA-induced hepatotoxicity ${ }^{[4]}$.

PA-HSOS is a rare disease with a low incidence. At present, there are limited reports about it. The classical triad of PA-HSOS consists of ascites, hepatomegaly, and increased bilirubin levels. The typical manifestations are swelling, damage and shedding of hepatic sinusoid endothelial cells in the hepatic acinus zone III and significant dilation and congestion of the hepatic sinusoids ${ }^{[5]}$. The clinical manifestations and test indicators of PA-HSOS are not specific. The diagnosis is mainly based on the history of ingestion of plants containing PAs and imaging examinations. This disease can be confused 
with Budd-Chiari syndrome (BCS), decompensated cirrhosis, or acute severe hepatitis, and misdiagnosis prevents prompt treatment. At present, there is no uniform standard for the diagnosis of PA-HSOS. The Baltimore and Seattle standards for hematopoietic stem cell transplantation (HSCT)-induced HSOS (HSCT-HSOS) are often used for reference. The Hepatobiliary Diseases Committee of the Chinese Society of Gastroenterology convened an expert consensus conference on the diagnosis and treatment of PAHSOS to evaluate current research in China and abroad. The "Nanjing criteria" developed by the committee to diagnose PA-HSOS include a confirmed history of PA-containing plant ingestion and the following: 1) abdominal distention and/or pain in the hepatic region, hepatomegaly and ascites, 2) elevation of serum total bilirubin levels or abnormal laboratory liver tests, and 3) evidence on enhanced CT or MRI, or pathological evidence that rules out other known causes of liver injury ${ }^{[6]}$. There is no specific treatment for PA-HSOS, and the mortality rate is high.

PA-HSOS is essentially a liver microcirculatory disorder, so anticoagulant, antithrombotic and microcirculation therapy can alleviate the progression of PA-HSOS. Studies have shown that anticoagulant therapy can significantly improve the cure rate of PA-HSOS patients $(P=0.004)^{[7]}$. PAHSOS patients with ascites and jaundice in the acute/subacute phase should receive anticoagulant therapy as soon as possible. At present, antifibrinogenic therapy is widely used in Western countries, and anticoagulation therapy is less well studied ${ }^{[8,9]}$.

Low molecular weight heparin in combination or sequence with warfarin are recommended as anticoagulant regimens in the "Nanjing criteria"[6]. Previous studies of heparin or low molecular weight heparin in the treatment of a small series of PA-HSOS patients in China reported recovery rates of $70.7 \%$ $88.9 \%{ }^{[5]}$. Low molecular weight heparin is significantly safer than unfractionated heparin. The recommended dose is $100 \mathrm{IU} / \mathrm{kg}$ every $12 \mathrm{~h}$, administered via subcutaneous injection and used with caution in patients with renal failure. Warfarin is a vitamin $\mathrm{K}$ inhibitor with a definite anticoagulant effect and low cost, but the therapeutic dose range is narrow, individual differences in response are large, and the effectiveness is susceptible to interactions with various foods and medicines. It carries a certain risk of bleeding. During treatment with warfarin, the international normalized ratio (INR) should be detected, and the dosage should be adjusted continuously. It needs to be combined with acid inhibitors. Patient compliance with warfarin therapy is poor.

A new oral anticoagulant (NOAC) rivaroxaban is the first direct oral factor Xa inhibitor. There is no need to monitor coagulation parameters, the anticoagulant effect is exact, and the bleeding risk is low. To some extent, it overcomes the shortcomings of traditional oral anticoagulants and has the characteristics of convenient administration, low bleeding risk, and good patient compliance. Rivaroxaban has been increasingly used as an alternative to warfarin for the prevention of thrombosis in patients with venous thromboembolism (VTE) and atrial fibrillation ${ }^{[10]}$. It has been widely used in the treatment of deep venous thrombosis and pulmonary embolism. The preventive dose is $10 \mathrm{mg}$ once a day, and the therapeutic dose is $15 \mathrm{mg}$ twice a day to $20 \mathrm{mg}$ twice a day. Rivaroxaban can also be used in the treatment of portal vein thrombosis (PVT) in patients with and without cirrhosis ${ }^{[10,11]}$. However, in the current research and 
guidelines, the anticoagulant treatment of venous thromboembolism of atypical location (VTE-AL), such as the spleen, kidney, mesentery and portal vein, is still dominated by low molecular weight heparin and warfarin.

The main adverse reaction associated with rivaroxaban is bleeding, and attention should be paid to the requirements for liver and kidney function. It is generally believed that the use of rivaroxaban in patients with moderate to severe renal insufficiency needs to be limited. The application of rivaroxaban in patients with PA-HSOS has not yet been reported. This study was a single-center retrospective case-control study designed to evaluate the efficacy and safety of rivaroxaban for the treatment of PA-HSOS.

Ethics, consent and permissions $\bigotimes$ This study was approved by the Ethics Committee of the First Affiliated Hospital of China Medical University.All participants included signed informed consent.

Consent to publish: We have obtained consent to publish from the participant to report individual patient data.

\section{Methods}

\section{Subjects}

The study included PA-HSOS patients in the acute/subacute phase who visited the Department of Gastroenterology, First Affiliated Hospital of China Medical University from 2017 to 2018. The inclusion criteria were as follows: 1 ) the diagnosis of PA-HSOS in the acute/subacute phase conformed to the "Nanjing criteria", 2) age older than 18 years, no genetic relationship with another enrolled patient, and 3) agree to participate in drug research and sign the informed consent. The exclusion criteria were as follows: 1) have a history of alcohol consumption, medication use or other liver diseases such as hepatitis, 2) have a history of other chronic diseases affecting liver function, 3) have contraindications for receiving anticoagulant drugs, and 4) do not consent to participate in drug research.

\section{Data}

The following information was collected from the enrolled patients: age, gender, clinical manifestations, history of taking Gynura segetum, disease history, and relevant laboratory indicators and examinations such as alanine aminotransferase (ALT) levels, aspartate aminotransferase (AST) levels, gammaglutamyltransferase (GGT) levels, alkaline phosphatase (ALP) levels, albumin (ALB) levels, total bilirubin (TBIL) levels, direct bilirubin (DBIL) levels, platelet (PLT) counts, prothrombin activity (PTA), D-D levels, liver stiffness, and enhanced CT to detect elasticity and vascular stenosis.

\section{Management}


All patients stopped taking Gynura segetum immediately after admission. They were given symptomatic supportive treatment such as liver protection, diuresis therapy, improvement of microcirculation, and intermittent plasma and albumin transfusions. Those with massive ascites underwent intermittent abdominal puncture and ascites drainage. Low molecular weight heparin 4100 iu was injected subcutaneously every $12 \mathrm{~h}$ for 2 weeks in all patients. The initial dosage of warfarin was $1.25 \mathrm{mg} /$ day in 10 patients. The INR was monitored $2-3$ days later, and the warfarin dosage was adjusted to maintain the INR at 2.0-3.0. Another 10 patients took $10 \mathrm{mg}$ of rivaroxaban orally once a day. Oral anticoagulation was discontinued when there was no intrahepatic vein stenosis and elasticity had recovered on imaging. All patients in the warfarin group took acid inhibitors at the same time to reduce the risk of gastrointestinal bleeding. The rivaroxaban group did not take acid inhibitors.

\section{Follow-up}

All patients were reexamined every two weeks or one month by liver ultrasound, liver elastography, routine blood tests, liver function tests, ion levels, coagulation parameters, D-D levels and other indicators. The other therapeutic drugs, such as liver protective treatments, were adjusted according to the improvement of clinical symptoms and biochemical test results. After symptoms disappeared and liver function returned to normal, liver protection therapy was discontinued. The length of the course of anticoagulation was decided according to whether there were vascular stenosis and elasticity on imaging. Rivaroxaban was stopped after patients had normal elasticity and hepatic vascular patency. Follow-up continued for 1 year after the condition was completely relieved or the patient died. Safety was assessed based on the occurrence of bleeding events in patients, including CRNMB, major bleeding, and major bleeding and CRNMB.

\section{Statistical analysis}

Normally distributed continuous variables are represented as the means \pm standard deviation; otherwise, the data are described as the medians (interquartile ranges). Count data are expressed as numbers and percentages. Independent $t$ tests were used for comparisons of continuous data, and the Mann-Whitney $U$ test was used for data with a skewed distribution. The Chi-square test or Fisher's exact test was used for comparisons of count data. $\mathrm{P}<0.05$ was considered statistically significant. All statistical analyses were performed with IBM SPSS 20.0.

\section{Results}

\section{Patient characteristics}

The study included 28 patients and excluded 4 patients with a previous history of alcohol consumption, 2 patients without follow-up data, and 2 patients with incomplete data; eventually, 20 patients were 
enrolled, with 10 patients in the warfarin group and 10 patients in the rivaroxaban group. All patients ranged from 43 to 77 years old, with 7 males and 13 females. The patients were mainly taking Gynura segetum due to low back pain, general health care, and other reasons. The daily dose was approximately $5 \mathrm{~g} 30 \mathrm{~g}$, and the continuous duration during which it had been taken was 2 weeks to 2 years. There were 2 patients with hypertension and 2 patients with diabetes mellitus in both groups; furthermore, there was 1 patient with a hepatic hemangioma in the rivaroxaban group, and 1 patients with a hepatic cyst in the warfarin group. In both groups, the hepatic parenchyma showed characteristic "map-like" and "patchy" nonuniform enhancement in the venous phase, and in the balanced phase, the hepatic veins became thinner, the blood flow velocity slowed down and the elasticity increased significantly. All patients had ascites, and 2 patients each in the warfarin and rivaroxaban groups had umbilical vein opening. There were 2 patients with PVT in each group; 2 patients in the warfarin group and 1 patient in the rivaroxaban group had splenomegaly, while 1 patient in each group showed a decrease in liver volume and a disordered proportion of each lobe. The general characteristics of the study subjects are shown in Table 1 , and the comparison of the clinical data is shown in Table 2. There were no significant differences in age, sex, duration of taking Gynura segetum, biochemical indicators, or elasticity between the two groups $(P>0.05)$.

\section{Outcome and prognosis}

One patient in the warfarin group did not regularly undergo reexaminations of the INR after being discharged from the hospital. After taking the medicine for 40 days, a massive hemorrhage of the digestive tract and urinary tract occurred, which improved after stopping the medicine. The remaining patients underwent regular reexaminations of their INRs. Four patients had CRNMB, which manifested as gingival bleeding, epistaxis, and skin ecchymosis; this improved after dose reduction, and no other serious adverse reactions occurred. Six patients in the rivaroxaban group had limb itching and needle-like pain approximately one week after initiating treatment. The patients were asked to take the medicine orally every other day, and all of the symptoms were relieved. Three patients presented with CRNMB, which manifested as gingival bleeding, epistaxis and skin ecchymosis; all symptoms improved after dose reduction, and no severe adverse reactions occurred. One patient in each group developed hepatic failure and hepatic encephalopathy during treatment and died after leaving the hospital. The remaining patients were discharged after clinical remission and cured after regular reexamination and therapy. No patients underwent interventional therapy. The recanalization rate of hepatic vessels, the incidence of hemorrhage and mortality during treatment are compared in Table 3 . There was no difference in the anticoagulant effect between the rivaroxaban group and warfarin group, but the treatment course of the rivaroxaban group was shorter, no additional acid inhibitor was needed, and the patients' compliance was better.

\section{Discussion}

In this study, we can see that rivaroxaban is effective for the treatment of PA-HSOS. The majority of patients with PA-HSOS experienced relief of their symptoms after approximately one month. Ultrasound 
showed blood flow in the three hepatic veins, and the blood test indexes returned to normal or nearly normal. During anticoagulation, vaginal bleeding, bloody ascites and other bleeding tendencies appeared in some patients, all of which improved after dose reduction, and no serious adverse events occurred due to bleeding. There was no significant difference in the incidence of bleeding events between the rivaroxaban group and warfarin group, which may be related to the small sample size. The duration of anticoagulation therapy in the warfarin group was longer than that in the rivaroxaban group, and the difference was statistically significant.

A study ${ }^{[12]}$ in 2015 assessed for the first time the difference in the risk of bleeding between traditional anticoagulants and direct oral anticoagulants (DOACs) in patients with liver cirrhosis. Thirty-nine patients with liver cirrhosis with CTP grades A and B were enrolled in the study. They were divided into the traditional anticoagulation (warfarin and low molecular weight heparin) group and the DOAC (factor Xa inhibitors: apisaban and rivaroxaban) group. The results showed that there was no significant difference in the risk of bleeding between the two groups $(P=0.9)$. Similar conclusions were drawn from a subsequent study ${ }^{[13]}$. Animal studies showed that rivaroxaban could help to alleviate hepatocyte fibrosis and reduce portal pressure in mice ${ }^{[14-16]}$, which provided support for the application of rivaroxaban in patients with liver cirrhosis.

In recent years, research on rivaroxaban for the treatment of VTE-AL has gradually increased. A study showed that the VTE recurrence and bleeding rates for NOACs used for VTE-AL are not different from those in patients with VTE-TL and are similar to those for enoxaparin ${ }^{[17]}$. Studies on PVT show that rivaroxaban is effective in the treatment of PVT with a low risk of bleeding and is superior to traditional oral drugs ${ }^{[11,18,19]}$. At present, there are few studies on the application of rivaroxaban in patients with liver cirrhosis and liver dysfunction. It is generally believed that patients with liver diseases at risk of coagulation abnormalities and clinical-related bleeding, including patients with liver cirrhosis CTP grade B and $\mathrm{C}$, should not use rivaroxaban.

To date, the application of rivaroxaban for the treatment of PA-HSOS has not been reported. Referring to the application of rivaroxaban in PVT and other abdominal organ venous embolism diseases, this study innovatively applied low molecular weight heparin in sequence with rivaroxaban oral treatment of PAHSOS. Considering the abnormal liver function and coagulation function of these patients, they were given a preventive dosage of $10 \mathrm{mg}$ once a day. The results showed that there was no difference in the anticoagulant effect between the rivaroxaban group and warfarin group, but the treatment course of the rivaroxaban group was shorter, no additional acid inhibitor was needed, and the patients' compliance was better.

In summary, the main reasons PA-HSOS patients take Gynura segetum are general health care and the treatment of lumbago and leg pain. Most patients experienced an onset of the adverse reaction within one month after taking Gynura segetum. More patients were females than male. Laboratory examinations of PA-HSOS are not specific. Imaging examinations play an important role in the diagnosis of PA-HSOS ${ }^{[20-22]}$. At present, there is no effective treatment for PA-HSOS. Early detection and active 
treatment can greatly reduce the mortality rate of patients. The clinical manifestations and examinations of the patients included in this study were typical, with a clear diagnosis and representative results.

A limitation of this study is that it was a single-center study, with few patients and no randomization or blinding. The number of included patients was small, and there is a lack of long-term follow-up data on treatment effects; therefore, further large-sample studies are needed. The replacement of warfarin anticoagulant therapy with rivaroxaban for PA-HSOS has greatly improved the safety of treatment and provided new methods for the diagnosis and treatment of the disease. The application of anticoagulant therapy in patients with PA-HSOS is still being explored. The clinical application of rivaroxaban anticoagulant therapy in patients with PA-HSOS, especially in patients with decompensated CTP grade C cirrhosis, still needs caution.

\section{Declarations}

\section{Acknowledgements}

The authors extend gratitude to all the enrolled patients for their contributions.

\section{Author contributions}

$\mathrm{HB}$ and $\mathrm{DL}$ are the first authors, $\mathrm{BC}$ and $\mathrm{YL}$ are corresponding authors. $\mathrm{BC}$ and $\mathrm{YL}$ carried out clinical studies; XH, JT, YW, RA and NW carried out data collection; HB and DL carried out data analysis and manuscript writing.

\section{Funding}

This work was supported by the NSFC (Natural Science Foundation of China) (No.81700453.)The funding body has no role in the design of the study and collection, analysis and interpretation of data and in writing the manuscript.

\section{Availability of data and materials}

The datasets supporting the conclusions of the current study are available at First Affiliated Hospital of China Medical University, which are available from the corresponding author on reasonable request.

\section{Ethics approval and consent to participate}

This study has been approved by the Ethics Committee of Ethics Committee of the First Affiliated Hospital of China Medical University, and written informed consents were obtained from all enrolled patients. 


\section{Consent for publication}

Not applicable.

\section{Competing interests}

The authors declare that they have no competing interests.

\section{Author details}

Department of Gastroenterology, First Affiliated Hospital of China Medical University, Shenyang 110001, Liaoning Province, China.

Corresponds to: Bing Chang, Associate Professor, Department of Gastroenterology, First Affiliated Hospital of China Medical University, Shenyang, Liaoning, China. cb000216@163.com; Yiling Li, Professor, Department of Gastroenterology, First Affiliated Hospital of China Medical University, Shenyang, Liaoning, China. lyl-72@163.com

\section{References}

1 Chen L, Mulder PPJ, Louisse J, et al. Risk assessment for pyrrolizidine alkaloids detected in (herbal) teas and plant food supplements. Regul Toxicol Pharmacol. 2017; 86: 292-302.

2 Lin G, Wang JY, Li N, et al. Hepatic sinusoidal obstruction syndrome associated with consumption of Gynura segetum. J Hepatol. 2011; 54: 666-673.

3 Wang X, Qi X, Guo X. Tusanqi-Related Sinusoidal Obstruction Syndrome in China: A Systematic Review of the Literatures. Medicine. 2015; 94: e942.

$4 \mathrm{Ma}$ J, Xia Q, Fu PP, et al. Pyrrole-protein adducts-A biomarker of pyrrolizidine alkaloid-induced hepatotoxicity. J Food Drug Anal. 2018; 26: 965-972.

5 Zhuge Y, Liu Y, Xie W, et al. Expert consensus on the clinical management of pyrrolizidine alkaloidinduced hepatic sinusoidal obstruction syndrome. J Gastroenterol Hepatol. 2019; 34: 634-642.

6 Cooperative group for hepatic and gall diseases, Chinese society of gastroenterology, Chinese medical association. Expert consensus on the diagnosis and treatment of pyrrole alkaline-related hepatic sinus obstruction syndrome. Chin J Dig. 2017; 37: 513-522. (in Chinese)

7 Wang Y, Qiao D, Li Y, et al. Risk factors for hepatic veno-occlusive disease caused by Gynura segetum: a retrospective study. BMC Gastroenterology. 2018; 18: 156. 
8 Kernan NA, Grupp S, Smith AR, et al. Final results from a defibrotide treatment-IND study for patients with hepatic veno-occlusive disease/sinusoidal obstruction syndrome. $\mathrm{Br} J$ Haematol. 2018; 181: 816827.

9 Kernan NA, Richardson PG, Smith AR, et al. Defibrotide for the treatment of hepatic veno-occlusive disease/sinusoidal obstruction syndrome following nontransplant-associated chemotherapy: Final results from a post hoc analysis of data from an expanded-access program. Pediatric Blood \& Cancer. 2018; 65: e27269.

10 Priyanka P, Kupec JT, Krafft M, et al. Newer Oral Anticoagulants in the Treatment of Acute Portal Vein Thrombosis in Patients with and without Cirrhosis. Int J Hepatol. 2018; 2018: 8432781.

11 Nery F, Valadares D, Morais S, et al. Efficacy and Safety of Direct-Acting Oral Anticoagulants Use in Acute Portal Vein Thrombosis Unrelated to Cirrhosis. Gastroenterology Res. 2017; 10: 141-143.

12 Intagliata NM, Henry ZH, Maitland H, et al. Direct Oral Anticoagulants in Cirrhosis Patients Pose Similar Risks of Bleeding When Compared to Traditional Anticoagulation. Dig Dis Sci. 2016; 61: 17211727.

13 Hum J, Shatzel JJ, Jou JH, et al. The efficacy and safety of direct oral anticoagulants vs traditional anticoagulants in cirrhosis. Eur J Haematol. 2017; 98: 393-397.

14 Mahmoud NI, Messiha BAS, Abo-Saif AA, et al. Inhibition of activated factor X; a new pathway in ameliorating carbon tetrachloride-induced liver fibrosis in rats. J Biochem Mol Toxicol. 2019; 4:e22287.

15 Dhar A, Sadiq F, Anstee Q M, et al. Thrombin and factor Xa link the coagulation system with liver fibrosis. BMC Gastroenterology. 2018; 18: 60.

16 Vilaseca M, García-Calderó H, Lafoz E, et al. The anticoagulant rivaroxaban lowers portal hypertension in cirrhotic rats mainly by deactivating hepatic stellate cells. Hepatology. 2017; 65: 2031-2044.

17 Janczak DT, Mimier MK, McBane R D, et al. Rivaroxaban and Apixaban for Initial Treatment of Acute Venous Thromboembolism of Atypical Location. Mayo Clin Proc. 2018; 93: 40-47.

18 Yang H, Kim S R, Song M J. Recurrent acute portal vein thrombosis in liver cirrhosis treated by rivaroxaban. Mayo Clin Proc. 2016; 22: 499-502.

19 Hanafy AS, Abd-Elsalam S, Dawoud MM. Randomized controlled trial of rivaroxaban versus warfarin in the management of acute non-neoplastic portal vein thrombosis. Vascul Pharmacol. 2019;113: 86-91.

20 Zhou H, Wang YJ, Lou H, et al. Hepatic Sinusoidal Obstruction Syndrome Caused by Herbal Medicine: CT and MRI Features. Korean J Radiol. 2014;15: 218-25. 
$21 \mathrm{Shao} \mathrm{H}$, Chen HZ, Zhu JS, et al. Computed tomography findings of hepatic veno-occlusive disease caused by Sedum aizoon with histopathological correlation. Braz J Med Biol Res. 2015; 48: 1145-50.

22 Kan X, Ye J, Rong X, et al. Diagnostic performance of Contrast-enhanced CT in Pyrrolizidine Alkaloidsinduced Hepatic Sinusoidal Obstructive Syndrome. Sci Rep. 2016; 6: 37998.

\section{Tables}

Table 1 Comparison of the general characteristics of the two groups

\begin{tabular}{|c|c|c|c|}
\hline & $\begin{array}{l}\text { Warfarin } \\
\text { group } \\
(\mathrm{N}=10)\end{array}$ & $\begin{array}{l}\text { Rivaroxaban } \\
\text { group } \\
(\mathrm{N}=10)\end{array}$ & $P$ \\
\hline \multicolumn{4}{|l|}{ Source of Gynura seqetum } \\
\hline Self-planted & 6 & 7 & 0.648 \\
\hline $\begin{array}{l}\text { Bought at a pharmacy or online } \\
\text { Method of consuming Gvnura segetum }\end{array}$ & 4 & 3 & 0.648 \\
\hline Grind into powder and flush & 2 & 2 & \\
\hline Boil in water & 4 & 5 & 0.661 \\
\hline Soak in water & 3 & 2 & 0.615 \\
\hline Soak in wine & 1 & 1 & \\
\hline \multicolumn{4}{|l|}{ First symptoms } \\
\hline Abdominal distention & 2 & 2 & \\
\hline $\begin{array}{l}\text { Abdominal distension, fatigue, poor } \\
\text { acceptance }\end{array}$ & 5 & 4 & 0.661 \\
\hline $\begin{array}{l}\text { acceptance } \\
\text { Abdominal distension, abdominal pain }\end{array}$ & 2 & 3 & 0.615 \\
\hline Fatigue, emaciation & 1 & 1 & \\
\hline \multicolumn{4}{|l|}{ Positive signs on physical examination } \\
\hline Abdominal bulging and mobile voiced sound & 10 & 10 & \\
\hline Upper abdominal tenderness & 2 & $\begin{array}{l}3 \\
2\end{array}$ & 0.615 \\
\hline $\begin{array}{l}\text { Edema of lower limbs } \\
\text { Jaundice }\end{array}$ & $\begin{array}{l}2 \\
3\end{array}$ & $\begin{array}{l}2 \\
2\end{array}$ & 0.615 \\
\hline \multicolumn{4}{|l|}{$\begin{array}{l}\text { Subcostal paipable spleen } \\
\text { Ascites level }\end{array}$} \\
\hline Light & 2 & 1 & 0.542 \\
\hline Moderate & 6 & 7 & 0.648 \\
\hline Severe & 2 & 2 & \\
\hline \multicolumn{4}{|l|}{ Anamnesis } \\
\hline Hypertension & 2 & 2 & 1 \\
\hline Diabetes & 2 & 2 & 1 \\
\hline Hepatic hemangioma or cyst & 1 & 1 & 1 \\
\hline
\end{tabular}

Table 2 Comparison of clinical data between the two groups 


\begin{tabular}{|c|c|c|c|c|}
\hline Item & $\begin{array}{l}\text { Warfarin group } \\
(\mathrm{N}=10)\end{array}$ & $\begin{array}{l}\text { Rivaroxaban } \\
\text { group } \\
(\mathrm{N}=10)\end{array}$ & Statistic & $P$ \\
\hline Age & $60.50 \pm 10.26$ & $60.20 \pm 11.03$ & $t=0.063$ & 0.95 \\
\hline Male/female & $4 / 6$ & $3 / 7$ & $\chi 2=0$ & 1 \\
\hline $\begin{array}{l}\text { Duration of taking Gynura segetum } \\
\text { (months) }\end{array}$ & $3.25[0.88,12.00]$ & $1.25[0.50,7.50]$ & $\mathrm{U}=44$ & 0.684 \\
\hline $\mathrm{ALT}(\mathrm{U} / \mathrm{L})$ & $93.00[24.50,244.25]$ & $45.00[19.25,174.75]$ & $\mathrm{U}=39$ & 0.436 \\
\hline AST (U/L) & $\begin{array}{l}190.50[57.50 \\
270.25]\end{array}$ & $61.50[49.00,239.00]$ & $\mathrm{U}=36.5$ & 0.315 \\
\hline ALP (U/L) & $\begin{array}{l}154.50[67.25 \\
202.25]\end{array}$ & $\begin{array}{l}140.00[109.00 \\
228.25]\end{array}$ & $\mathrm{U}=62$ & 0.393 \\
\hline GGT (U/L) & $\begin{array}{l}215.00[109.25 \\
342.75]\end{array}$ & $113.00[69.75,356.25]$ & $\mathrm{U}=44$ & 0.684 \\
\hline TBIL $(\mu \mathrm{mol} / \mathrm{L})$ & $47.70[29.30,61.63]$ & $33.95[21.75,43.28]$ & $\mathrm{U}=32$ & 0.19 \\
\hline DBIL $(\mu \mathrm{mol} / \mathrm{L})$ & $22.55[7.55,32.05]$ & $19.65[12.63,26.60]$ & $\mathrm{U}=49$ & 0.971 \\
\hline ALB $(g / L)$ & $27.20[24.68,30.28]$ & $27.95[26.30,30.98]$ & $\mathrm{U}=59.5$ & 0.481 \\
\hline $\mathrm{PT}(\mathrm{s})$ & $16.70[15.05,19.18]$ & $18.05[15.73,19.13]$ & $\mathrm{U}=55$ & 0.739 \\
\hline $\mathrm{Fg}(\mathrm{g} / \mathrm{L})$ & $1.88[1.64,2.92]$ & $2.74[1.69,3.81]$ & $\mathrm{U}=63.5$ & 0.315 \\
\hline PTA (\%) & $60.20 \pm 14.58$ & $60.30 \pm 13.53$ & $t=0.015$ & 0.988 \\
\hline INR & $1.49 \pm 0.26$ & $1.44 \pm 0.20$ & $t=0.427$ & 0.674 \\
\hline $\mathrm{D}-\mathrm{D}(\mu \mathrm{g} / \mathrm{mL})$ & $3.10 \pm 1.65$ & $3.07 \pm 1.94$ & $t=0.038$ & 0.970 \\
\hline $\operatorname{PLT}(10 \sim 9 / \mathrm{L})$ & $\begin{array}{l}105.50[81.00 \\
211.00]\end{array}$ & $\begin{array}{l}123.50[111.00 \\
158.25]\end{array}$ & $\mathrm{U}=63$ & 0.353 \\
\hline Hepatic elasticity $(\mathrm{kPa})$ & $41.85[34.10,53.33]$ & $38.15[26.70,55.93]$ & $\mathrm{U}=46$ & 0.796 \\
\hline Anticoagulation course (days) & $90.00[90.00,90.00]$ & $30.00[30.00,90.00]$ & $\mathrm{U}=15$ & 0.007 \\
\hline Hospitalization time (days) & $22.00[14.75,24.00]$ & $18.50[14.00,25.25]$ & $\mathrm{U}=46$ & 0.796 \\
\hline
\end{tabular}

Table 3 Comparison of hepatic vascular recanalization rates, hemorrhagic events and mortality rates between the two groups

\begin{tabular}{llll}
\hline & $\begin{array}{l}\text { Warfarin } \\
\text { group } \\
(\mathrm{N}=10)\end{array}$ & $\begin{array}{l}\text { Rivaroxaban } \\
\text { group } \\
(\mathrm{N}=10)\end{array}$ & $P$ \\
\hline 1-month recanalization rate & $40 \%$ & $60 \%$ & 0.39 \\
2-month recanalization rate & $60 \%$ & $70 \%$ & 0.65 \\
3-month recanalization rate & $90 \%$ & $90 \%$ & 1 \\
Incidence of all bleeding events & 5 & 3 & 0.37 \\
Incidence of massive hemorrhage & $10 \%$ & $0 \%$ & 0.32 \\
Incidence of CRNMB & $40 \%$ & $30 \%$ & 0.65 \\
Incidence of massive hemorrhage with & $0 \%$ & $0 \%$ & 1 \\
CRNMB & $10 \%$ & $10 \%$ & 1 \\
Fatality rate & & & \\
\hline
\end{tabular}

